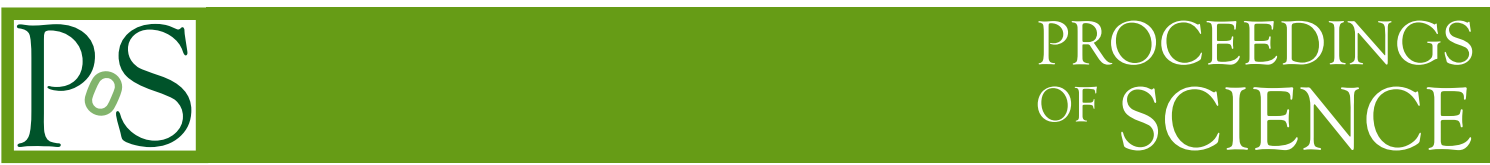

\title{
Three-quark systems in MA and MC projected QCD
}

\author{
Hideaki lida* \\ Yukawa Institute for Theoretical Physics, Kyoto University \\ Kitashirakawaoiwake, Sakyo, Kyoto 606-8502, Japan \\ E-mail: iida@yukawa.kyoto-u.ac.jp
}

\section{Naoyuki Sakumichi and Hideo Suganuma}

Department of Physics, Kyoto University,

Kitashirakawaoiwake, Sakyo, Kyoto 606-8502, Japan

E-mail: sakumichi@scphys.kyoto-u.ac.jp.

suganuma@scphys.kyoto-u.ac.jp

We study three quark systems in Maximally Abelian (MA) and Maximal Center (MC) projected QCD on quenched SU(3) lattice, and also in the monopole/photon part, where only the colorelectric/magnetic current exists, using the Hodge decomposition. First, we perform the quantitative study of the three-quark (3Q) potential $V_{3 \mathrm{Q}}$ and the string tension $\sigma_{3 \mathrm{Q}}$ in baryons. For MA projected QCD, the monopole part and MC projected QCD, we find that the confinement potential in $V_{3 \mathrm{Q}}$ obeys the Y-Ansatz and the string tension $\sigma_{3 \mathrm{Q}}$ is approximately equal to that in $\mathrm{SU}(3) \mathrm{QCD}$. The universality of the string tension, $\sigma_{3 \mathrm{Q}} \simeq \sigma_{\mathrm{QQ}}$, is also found between the $3 \mathrm{Q}$ and the $\mathrm{QQ}$ potentials. We find a strong similarity of the inter-quark potential between the monopole part and MC projected QCD. In contrast, almost no confinement force is found in the inter-quark potential in the photon part. Next, we study the spectrum of light hadrons in MA projected QCD and the monopole/photon part, paying attention to the $\mathrm{N}-\Delta$ mass splitting. We find that the $\mathrm{N}-\Delta$ mass splitting is significantly reduced in MA projected QCD and the monopole part, where the one-gluon-exchange effect or the Coulomb-potential part is largely reduced due to the Abelianization or the Hodge decomposition. This fact seems to indicate that the main origin of the mass splitting is one-gluon exchange.

The XXVI International Symposium on Lattice Field Theory

July 14 - 19, 2008

Williamsburg, Virginia, USA

\footnotetext{
${ }^{*}$ Speaker.
} 


\section{Introduction}

Color confinement in QCD is one of the most challenging problems in physics. One of the possible scenarios of color confinement is the dual-superconductor picture, proposed by Nambu [1], 't Hooft and Mandelstam in 70's. The key point of color confinement is one-dimensional squeezing of the color-electric flux, and the dual-superconductor picture realizes such squeezing due to the dual Meissner effect, which is the dual version of the Meissner effect in superconductors.

However, there are two large gaps between the dual-superconductor picture and QCD. One gap is the non-Abelian nature of QCD. While QCD is a non-Abelian gauge theory, the dualsuperconductor picture is based on the Abelian gauge theory subject to the Maxwell-type equation, where electro-magnetic duality is manifest. The other gap is the existence of the monopole degrees of freedom. The dual-superconductor picture requires condensation of color-magnetic monopoles as the key concept, while QCD does not have such a monopole as the elementary degrees of freedom. To compensate these two gaps, Maximally Abelian (MA) gauge is a suitable gauge choice [2]. The important point is that the off-diagonal gluons have a large effective mass of about $1 \mathrm{GeV}$ in MA gauge [3]. Therefore, only the diagonal gluons are relevant in infrared region in MA gauge. The other important point is that color-magnetic monopoles appear as topological objects reflecting the nontrivial homotopy group: $\Pi_{2}\left(\mathrm{SU}\left(N_{c}\right) / \mathrm{U}(1)^{N_{c}-1}\right)=\mathbf{Z}_{\infty}^{N_{c}-1}$. Therefore, QCD is reduced to an Abelian theory with monopoles in MA gauge in infrared region. In the following, we call the Abelian theory as "MA projected QCD".

MA projected QCD is decomposed into two parts by the Hodge decomposition. One is "monopole part", where the color-magnetic monopole current $k_{\mu}$ exists without the color-electric current $j_{\mu}$. The other is "photon part", where the color-electric current $j_{\mu}$ exists without the colormagnetic current $k_{\mu}$. Since monopoles are considered to contribute the nonperturbative phenomena such as confinement, roughly speaking, the monopole part is responsible for "nonperturbative" phenomena related to the vacuum structure of QCD, and the photon part is responsible for "perturbative" phenomena. Actually, the QQ potential can be well separated into the linear confinement potential in the monopole part and the Coulomb potential in the photon part by the Hodge decomposition. Moreover, the chiral symmetry is spontaneously broken and instantons survive in the monopole part, while no spontaneous chiral symmetry breaking appears and no instanton survives in the photon part [4, 5]. Then, using the monopole/photon decomposition, we can clarify whether each QCD phenomenon mainly originates from "nonperturbative" nature or "perturbative" nature.

Similar essence of color confinement can be also extracted in Maximal Center (MC) gauge [6], which is a gauge choice that extracts the degrees of freedom of the center group $Z_{3}$ of QCD. A four-dimensional $\mathrm{Z}_{3}$-spin system can be obtained through $\mathrm{MC}$ projection after $\mathrm{MC}$ gauge fixing, and the linear confinement potential can be well extracted as the QQ potential in this system [6].

Recently, the detailed analysis of the three-quark (3Q) potential was performed in lattice QCD [7], which reveals the Y-type linear confinement [7] and the Y-shaped flux-tube formation in baryons [8]. In this paper, aiming to clarify the quark confinement mechanism in baryons, we perform the quantitative lattice study of the $3 \mathrm{Q}$ potential in MA projected QCD, the monopole/photon part [9] and MC projected QCD. (The semi-quantitative study of the 3Q potential was done in MA gauge in Ref.[8].) We also study the spectrum of light hadrons, i.e., $\mathrm{N}, \Delta, \pi$ and $\rho$, in MA projected $\mathrm{QCD}$ and the monopole/photon part, paying attention to the $\mathrm{N}-\Delta$ mass splitting. 


\section{MA gauge fixing, MA projection, Hodge decomposition and MC gauge fixing}

In lattice QCD, Maximally Abelian (MA) gauge is defined so as to maximize the quantity $R_{\mathrm{MA}}\left[U_{\mu}(s)\right] \equiv \operatorname{Re} \sum_{s, \mu} \operatorname{Tr}\left(U_{\mu}(s) \vec{H} U_{\mu}^{\dagger}(s) \vec{H}\right)$ by SU(3) gauge transformation, with the link-variable $U_{\mu}(s)$ and the Cartan subalgebra $\vec{H}$ of SU(3). We denote the MA gauge fixed configuration by $U_{\mu}^{\mathrm{MA}}(s) \in \mathrm{SU}(3)_{c}$. After the MA gauge fixing, we extract Abelian link-variable $u_{\mu}^{\mathrm{Abel}}(s) \in \mathrm{U}(1)^{2} \subset$ $\mathrm{SU}(3)_{c}$ from $U_{\mu}^{\mathrm{MA}}(s) \in \mathrm{SU}(3)_{c}$ by maximizing $\operatorname{Re} \operatorname{Tr}\left(u_{\mu}^{\mathrm{Abel}}(s) U_{\mu}^{\mathrm{MA}}(s)^{\dagger}\right)$. MA projection is defined by the replacement of the $\mathrm{SU}(3)$ link-variable $\left\{U_{\mu}^{\mathrm{MA}}(s)\right\}$ by the Abelian link-variable $\left\{u_{\mu}^{\mathrm{Abel}}(s)\right\}$.

In the MA projected QCD, there appear not only the electric current $j_{\mu}(s)$ but also the magnetic (monopole) current $k_{\mu}(s)$. By the Hodge decomposition, as will be explained below, the MA projected QCD can be decomposed into the "monopole part" which only includes the magnetic current $k_{\mu}$ and the "photon part" which only includes the electric current $j_{\mu}$. We define the gauge field $\theta_{\mu}^{i}(s) \in(-\pi, \pi](i=1,2,3)$ as $u_{\mu}^{\mathrm{Abel}}(s)=\operatorname{diag}\left(e^{i \theta_{\mu}^{1}}(s), e^{i \theta_{\mu}^{2}}(s), e^{i \theta_{\mu}^{3}}(s)\right)$. The field strength tensor $\theta_{\mu \nu}^{i}(s) \in(-\pi, \pi]$ is defined as $(\partial \wedge \theta)_{\mu \nu}^{i} \equiv \partial_{\mu} \theta_{\nu}^{i}-\partial_{\nu} \theta_{\mu}^{i}=\theta_{\mu \nu}^{i}+2 \pi n_{\mu \nu}^{i}$, with $n_{\mu \nu}^{i} \in \mathbf{Z}$. From $\theta_{\mu \nu}^{i}(s)$, the electric current $j_{v}^{i}(s)$ and the magnetic current $k_{v}^{i}(s)$ are defined as $j_{v}^{i} \equiv \partial_{\mu} \theta_{\mu \nu}^{i}$ and $k_{v}^{i} \equiv \partial_{\mu}^{*} \theta_{\mu \nu}^{i}=-2 \pi \partial_{\mu}^{*} n_{\mu \nu}^{i}$, with ${ }^{*} \theta_{\mu \nu}^{i} \equiv \varepsilon_{\mu \nu \rho \sigma} \theta_{\rho \sigma}^{i} / 2$. For simple notation, we hereafter abbreviate the index $i$. Since the photon part has only the electric current by definition, the field strength $\theta_{\mu \nu}^{\mathrm{Ph}}$ in the photon part obeys $j_{v}=\partial_{\mu} \theta_{\mu \nu}^{\mathrm{Ph}}$ and $\partial_{\mu}^{*} \theta_{\mu \nu}^{\mathrm{Ph}}=0$. Then, the gauge field $\theta_{\mu}^{\mathrm{Ph}}$ in the photon part obeys the four-dimensional Poisson equation $j_{v}(s)=\partial^{2} \theta_{v}^{\mathrm{Ph}}(s)$, by taking $\mathrm{U}(1)$ Landau gauge fixing, $\partial_{\mu} \theta_{\mu}^{\mathrm{Ph}}(s)=0$, for the residual $\mathrm{U}(1)^{2}$ gauge symmetry. Solving the Poisson equation, $\theta_{\mu}^{\mathrm{Ph}}(s)$ can be obtained. The gauge field $\theta^{\mathrm{Mo}}(s)$ in the monopole part is obtained as $\theta_{\mu}^{\mathrm{Mo}}(s)=\theta_{\mu}(s)-\theta_{\mu}^{\mathrm{Ph}}(s)$.

Maximal Center (MC) gauge fixing [6] is defined to maximize $\sum_{s, \mu}\left|\operatorname{Tr} U_{\mu}(s)\right|^{2}$ by gauge transformation, and the link-variable maximally approaches to $\mathrm{Z}_{3}$ element in $\mathrm{MC}$ gauge. After the MC gauge fixing, $\mathrm{Z}_{3}$ link-variable $z_{\mu}(s) \in \mathrm{Z}_{3}$ is extracted by maximizing $\operatorname{Re} \operatorname{Tr}\left(z_{\mu}(s) U_{\mu}(s)^{\dagger}\right)$, and MC projection is defined as the replacement by the $Z_{3}$ link-variable $z_{\mu}(s)=e^{2 \pi i m(s) / 3} \cdot \mathbf{1}(m(s)=0,1,2)$.

\section{The three-quark potential in MA projected QCD}

The three-quark (3Q) potential $V_{3 \mathrm{Q}}$ [7] is obtained as $V_{3 \mathrm{Q}}=-\lim _{T \rightarrow \infty} \frac{1}{T} \ln \left\langle W_{3 \mathrm{Q}}(T)\right\rangle$ from the 3Q Wilson loop $W_{3 \mathrm{Q}}(T) \equiv \frac{1}{3 !} \varepsilon_{a b c} \varepsilon_{a^{\prime} b^{\prime} c^{\prime}} U_{1}^{a a^{\prime}} U_{2}^{b b^{\prime}} U_{3}^{c c^{\prime}}$ with $U_{k} \equiv P \exp \left\{i g \int_{\Gamma_{k}} d x_{\mu} A^{\mu}(x)\right\}(k=1,2,3)$ as the path-ordered product along $\Gamma_{k}$ as shown in Fig. 1. We calculate the 3Q potential for 120 different patterns of 3Q systems in lattice QCD with $16^{3} \times 32$ and $\beta \equiv 6 / g^{2}=6.0$, i.e., $a \simeq 0.1 \mathrm{fm}$ for the lattice spacing. To enhance the ground-state component, we adopt the smearing method. For the calculation, we use 100 gauge configurations generated with NEC SX-8R in Osaka University.

We thus obtain the 3Q potential $V_{3 \mathrm{Q}}$ in $\mathrm{SU}(3) \mathrm{QCD}$, MA projected QCD, the monopole/photon part and MC projected QCD, respectively. For the quantitative analysis, we consider the functional fit for the obtained lattice data of the 3Q potential in each part using the Y-Ansatz [7],

$$
V_{3 \mathrm{Q}}=-A_{3 \mathrm{Q}} \sum_{i<j} \frac{1}{\left|\mathbf{r}_{i}-\mathbf{r}_{j}\right|}+\sigma_{3 \mathrm{Q}} L_{\min }+C_{3 \mathrm{Q}}=-A_{3 \mathrm{Q}} / L_{\mathrm{Coul}}+\sigma_{3 \mathrm{Q}} L_{\min }+C_{3 \mathrm{Q}}
$$

with the static quark location $\mathbf{r}_{i}(i=1,2,3), L_{\text {Coul }} \equiv\left(\sum_{i<j} \frac{1}{\left|\mathbf{r}_{i}-\mathbf{r}_{j}\right|}\right)^{-1}=(1 / a+1 / b+1 / c)^{-1}$ and $L_{\min } \equiv \mathrm{AP}+\mathrm{BP}+\mathrm{CP}$, the minimum length connecting the three quarks, as shown in Fig. 2. The 3Q potential in SU(3) QCD is known to be well fit by the Y-Ansatz within 1\%-level deviation [7]. 


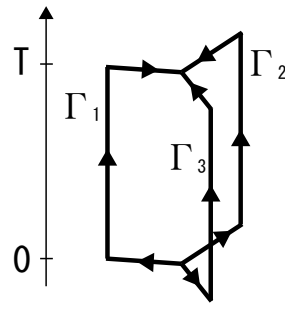

Figure 1: The schematic description of the three-quark Wilson loop $W_{3 Q}$.

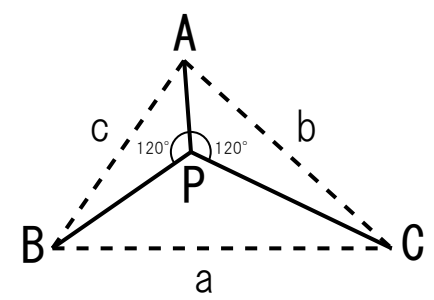

Figure 2: The system of static three quarks located at A, B and C. P denotes the Fermat point.

\section{Numerical results of the three-quark potential in MA and MC projected QCD}

Now, we show the numerical results of the three-quark (3Q) potential. Figure 3 and 4 show the 3Q potential in SU(3) QCD, MA projected QCD, the monopole part and MC projected QCD, plotted against $L_{\min }$. For MA projected QCD, the monopole part and MC projected QCD, we find that the $3 \mathrm{Q}$ potential $V_{3 \mathrm{Q}}$ is approximately a single-valued function of $L_{\mathrm{min}}$, although $V_{3 \mathrm{Q}}$ generally depends on three independent variables, e.g., $a, b$ and $c$. We note that the results in the monopole part and MC projected QCD are very similar apart from an irrelevant constant. Figure 5 and 6 show the 3Q potential in the photon part plotted against $L_{\min }$ and $L_{\mathrm{Coul}}$, respectively. The 3Q potential in the photon part is not a single-valued function of $L_{\min }$, but approximately a single-valued function of $L_{\text {Coul }}$ except for $L_{\text {Coul }} \leq 1$, where the discretization error is large.

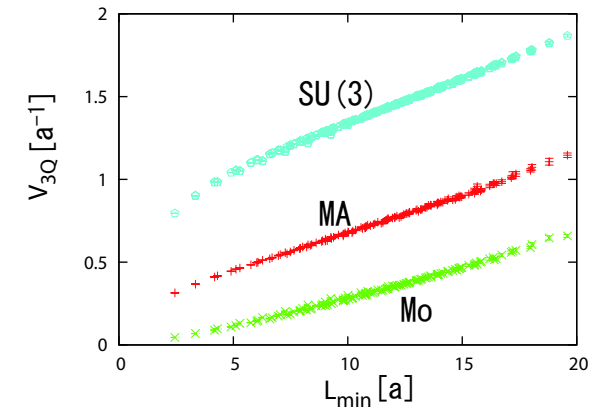

Figure 3: The 3Q potential $V_{3 \mathrm{Q}}$ in $\mathrm{SU}(3) \mathrm{QCD}$, MA projected QCD (MA) and the monopole part (Mo) plotted against $L_{\min }$.

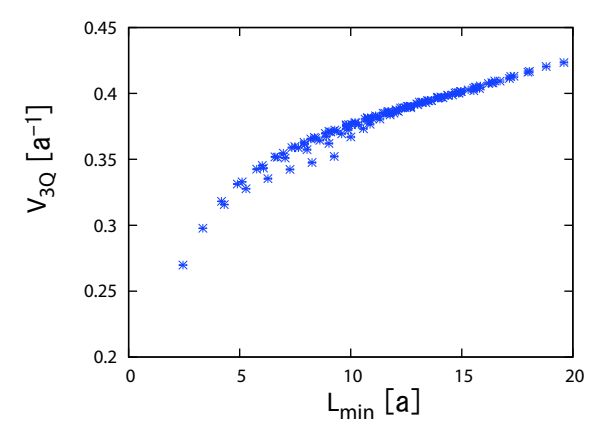

Figure 5: The 3Q potential $V_{3 Q}$ in the photon part plotted against $L_{\min }$.

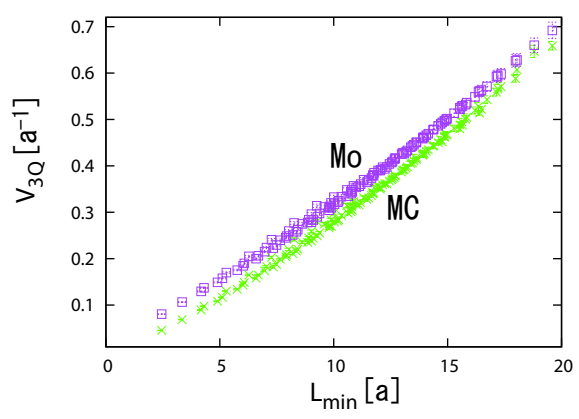

Figure 4: The $3 \mathrm{Q}$ potential $V_{3 \mathrm{Q}}$ in the monopole part (Mo) and MC projected QCD (MC) plotted against $L_{\min }$.

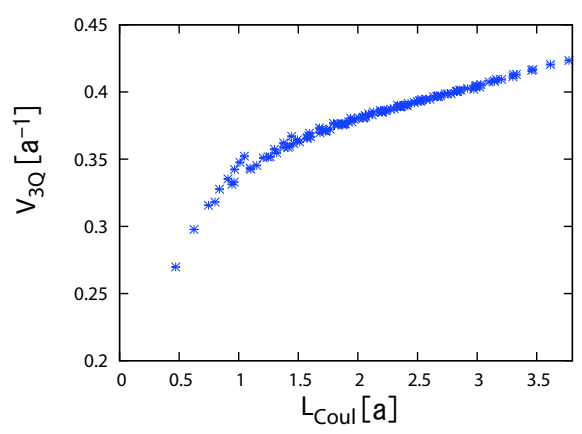

Figure 6: The $3 \mathrm{Q}$ potential $V_{3 \mathrm{Q}}$ in the photon part plotted against $L_{\text {Coul }}$. 
We next do the fit analysis by the Y-Ansatz, i.e., the functional form of Eq. (3.1). For the monopole part and $\mathrm{MC}$ projected QCD, short-distance lattice data are excluded in the fit analysis. For MA projected QCD, the monopole part and MC projected QCD, we find that the $3 \mathrm{Q}$ potential $V_{3 \mathrm{Q}}$ obeys the Y-Ansatz for the confinement potential and includes almost no Coulomb potential, which makes $V_{3 \mathrm{Q}}$ be a single-valued function of $L_{\min }$. The reduction of the Coulomb potential in MA projected QCD can be explained by the reduction of the gluon components from 8 to 2 through the Abelianization. On the other hand, the $3 \mathrm{Q}$ potential $V_{3 \mathrm{Q}}$ in the photon part includes almost no linear potential proportional to $L_{\mathrm{min}}$, which makes $V_{3 \mathrm{Q}}$ be a single-valued function of $L_{\mathrm{Coul}}$.

We summarize in Table 1 the string tension $\sigma_{3 \mathrm{Q}}$ in the $3 \mathrm{Q}$ potential and the string tension $\sigma_{\mathrm{QQ}}$ in the QQ potential for SU(3) QCD, MA projected QCD, the monopole part, MC projected QCD and the photon part. We find the approximate equality of the string tension among SU(3) QCD, MA projected QCD, the monopole part and MC projected QCD, as well as the universality of the string tension as $\sigma_{3 \mathrm{Q}} \simeq \sigma_{\mathrm{QQ}}$. In contrast, almost zero string tension emerges in the photon part.

\begin{tabular}{cccccc}
\hline \hline & SU(3) QCD & MA projected QCD & Monopole part & MC projected QCD & Photon part \\
\hline$\sigma_{3 \mathrm{Q}}$ & 0.046 & 0.0456 & 0.0382 & 0.0372 & $0.0021(\sim 0)$ \\
$\sigma_{\mathrm{QQ}}$ & 0.0506 & 0.0439 & 0.0402 & 0.0361 & $0.0041(\sim 0)$ \\
\hline \hline
\end{tabular}

Table 1: The string tension $\sigma_{3 \mathrm{Q}}$ in the $3 \mathrm{Q}$ potential and the string tension $\sigma_{\mathrm{QQ}}$ in the QQ potential in SU(3) $\mathrm{QCD}$, MA projected QCD, the monopole part, MC projected QCD and the photon part in the lattice unit.

\section{5. $\mathbf{N}-\Delta$ mass splitting in MA projected QCD}

In this section, we study the mass spectrum of light hadrons, $\pi, \rho$, nucleon $(\mathrm{N})$ and $\Delta$, in MA projected QCD. In particular, we focus on the $\mathrm{N}-\Delta$ mass splitting and its origin. In the ordinary constituent quark model, the $\mathrm{N}-\Delta$ mass splitting originates from the color-magnetic interaction [10]. As another possibility of its origin, instantons are proposed to contribute to the $\mathrm{N}-\Delta$ mass splitting [11]. The two explanations on the origin are largely different, because the color-magnetic interaction appears from the one-gluon-exchange process and is "perturbative", while the interaction caused by instantons is nonperturbative.

To clarify whether the main origin of the $\mathrm{N}-\Delta$ mass splitting is perturbative or nonperturbative, we utilize MA projected QCD and the monopole part, where the nonperturbative nature such as instantons almost survives [4, 5] but one-gluon-exchange effects are largely reduced due to the Abelianization and the Hodge decomposition. In fact, the small/large reduction of the $\mathrm{N}-\Delta$ mass splitting in the monopole part is expected to indicate the nonperturbative/perturbative origin.

Based on this strategy, we perform the lattice QCD calculation with Wilson quarks at $\beta=6.0$ $\left(a \simeq 0.1 \mathrm{fm}\right.$ ) on $16^{3} \times 32$. The used hopping parameter $\kappa$ and the critical hopping parameter $\kappa_{c}$ where the pion is massless are summarized in Table 2. We find that the value of $\kappa_{c}$ is significantly different among SU(3) QCD, MA projected QCD and the monopole part. Then, we compare the $\mathrm{N}-\Delta$ mass splitting among them in the chiral limit, where the physical situation is the same.

Figure 7 and 8 show the mass of $\mathrm{N}$ and $\Delta$ in MA projected QCD and the monopole part, respectively. The numerical results in the chiral limit are summarized in Table 3. The $\mathrm{N}-\Delta$ mass splitting is significantly reduced in MA projected QCD and the monopole part. Such reduction 


\begin{tabular}{cccc}
\hline \hline & $\kappa$ & $\kappa_{c}$ & Gauge configuration \\
\hline SU(3) QCD & $0.1520,0.1540,0.1550$ & 0.1571 & 100 \\
MA projected QCD & $0.1350,0.1365,0.1370,0.1380$ & 0.1422 & 250 \\
Monopole part & $0.1260,0.1270,0.1275,0.1282$ & 0.1392 & 250 \\
Photon part & $0.1260,0.1270,0.1275$ & - & 100 \\
\hline \hline
\end{tabular}

Table 2: The hopping parameter $\kappa$ used in the calculation and the critical hopping parameter $\kappa_{c}$, for SU(3) QCD, MA projected QCD, the monopole and the photon part. The gauge configuration number is also listed.

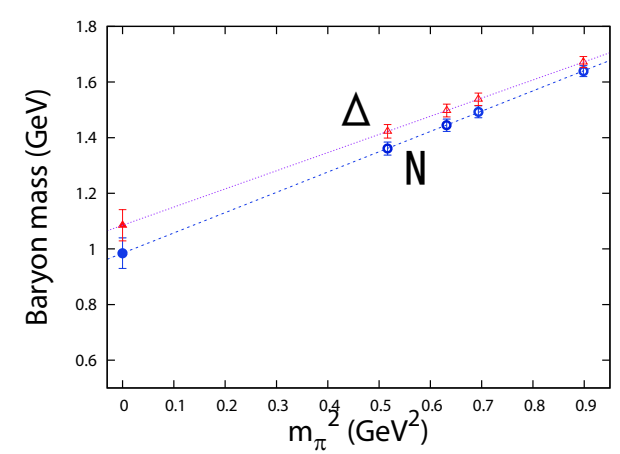

Figure 7: The mass of nucleon $(\mathrm{N})$ and $\Delta$ in MA projected QCD plotted against the pion-mass squared, $m_{\pi}^{2}$. The baryon mass in chiral limit is obtained by standard linear chiral extrapolation.

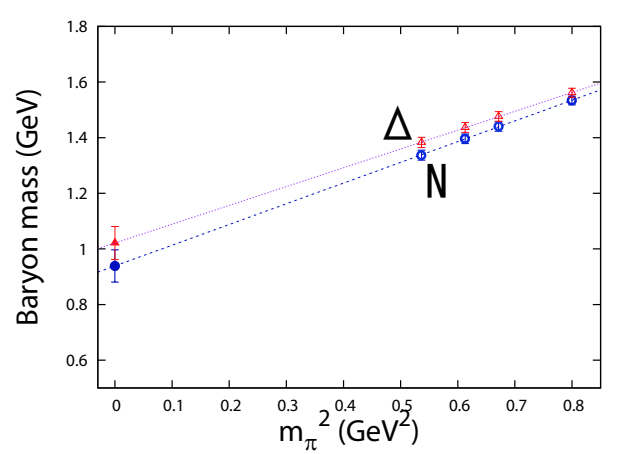

Figure 8: The mass of nucleon $(\mathrm{N})$ and $\Delta$ in the monopole part plotted against the pion-mass squared, $m_{\pi}^{2}$. The baryon mass in chiral limit is obtained by standard linear chiral extrapolation.

\begin{tabular}{cccc}
\hline \hline & SU(3) QCD & MA projected QCD & Monopole part \\
\hline $\mathrm{N}$ mass $[\mathrm{GeV}]$ & $1.058(20)$ & $0.985(55)$ & $0.939(58)$ \\
$\Delta$ mass $[\mathrm{GeV}]$ & $1.254(29)$ & $1.085(56)$ & $1.021(60)$ \\
$\mathrm{N}-\Delta$ mass splitting $[\mathrm{GeV}]$ & $\sim 0.20$ & $\sim 0.10$ & $\sim 0.08$ \\
\hline \hline
\end{tabular}

Table 3: The mass of nucleon $(\mathrm{N})$ and $\Delta$ in the chiral limit, for $\mathrm{SU}(3)$ QCD, MA projected QCD and the monopole part. The $\mathrm{N}-\Delta$ mass splitting is also shown. The jackknife error estimation is used.

can be explained by the reduction of the one-gluon-exchange effect, which is considered to be the origin of the $\mathrm{N}-\Delta$ mass splitting in the constituent quark model. Actually, the MA projection or the Hodge decomposition largely reduces one-gluon-exchange effects, which leads to the reduction of the Coulomb-potential part in the inter-quark potential, as was shown in the previous section. In fact, this result seems to indicate that the main origin of the $\mathrm{N}-\Delta$ mass splitting is the one-gluonexchange effect, which is consistent with the explanation by the constituent quark model.

In contrast, in the photon part, we observe the almost complete degeneracy between $\mathrm{N}$ and $\Delta$ and between $\pi$ and $\rho$ for each $\kappa$. The ratio of the baryon mass to the meson mass is 3 to 2 , which is the ratio of the quark number of the hadron. Moreover, all the hadrons are massless in the chiral limit, where the pion is massless. These facts indicate that, in the photon part, the system consists of three or two quasi-free quarks, and no compact bound state like hadrons is created. (We note however that the photon part is rather different from no interaction case with $U_{\mu}(s)=1$.) 


\section{Summary and conclusion}

We have studied the three-quark (3Q) potential quantitatively in MA and MC projected QCD on quenched SU(3) lattice. For SU(3) QCD, MA projected QCD, the monopole part and MC projected QCD, we have found the Y-Ansatz for the confinement potential in $V_{3 \mathrm{Q}}$ and the approximate equality of the string tension as $\sigma_{3 \mathrm{Q}}^{\mathrm{SU}(3)} \simeq \sigma_{3 \mathrm{Q}}^{\mathrm{MA}} \simeq \sigma_{3 \mathrm{Q}}^{\mathrm{Mo}} \simeq \sigma_{3 \mathrm{Q}}^{\mathrm{MC}}$. In each part, the universality of the string tension is found as $\sigma_{3 \mathrm{Q}} \simeq \sigma_{\mathrm{QQ}}$ between the $3 \mathrm{Q}$ and the $\mathrm{QQ}$ potentials. We have found the strong similarity of the inter-quark potential between monopole part and MC projected QCD. In contrast, almost no confinement force is found in the photon part, i.e., $\sigma_{3 \mathrm{Q}}^{\mathrm{Ph}} \simeq 0$.

We have also studied the light hadron mass spectrum in MA projected QCD, especially focusing on the $\mathrm{N}-\Delta$ mass splitting. The $\mathrm{N}-\Delta$ mass splitting is significantly reduced in MA projected QCD and the monopole part, where the one-gluon-exchange effect or the Coulomb-potential part is largely reduced due to the Abelianization or the Hodge decomposition. This behavior seems consistent with the constituent quark model, where the origin of the splitting is one-gluon exchange. In the photon part, the system consists of quasi-free quarks and compact hadrons are not created.

\section{References}

[1] Y. Nambu, Strings, Monopoles and Gauge Fields, Phys. Rev. D10 (1974) 4262; G. 't Hooft, Gauge Fields with Unified Weak, Electromagnetic, and Strong Interactions, (1975); S. Mandelstam, Vortices and Quark Confinement in Nonabelian Gauge Theories, Phys. Rept. C23 (1976) 245.

[2] G. 't Hooft, Topology of the Gauge Condition and New Confinement Phases in Nonabelian Gauge Theories, Nucl. Phys. B190 (1981) 455; Z. F. Ezawa and A. Iwazaki, Abelian Dominance and Quark Confinement in Yang-Mills Theories, Phys. Rev. D25 (1982) 2681.

[3] K. Amemiya and H. Suganuma, Off-Diagonal Gluon Mass Generation and Infrared Abelian Dominance in the Maximally Abelian Gauge in Lattice QCD, Phys. Rev. D60 (1999) 114509.

[4] O. Miyamura, Chiral Symmetry Breaking in Gauge Fields dominated by Monopoles on SU(2) Lattices, Phys. Lett. B353 (1995) 91.

[5] H. Suganuma, A. Tanaka, S. Sasaki and O. Miyamura, Evidence of Strong Correlation between Instanton and QCD-Monopole on SU(2) Lattice, Nucl. Phys. Proc. Suppl. B47 (1996) 302.

[6] L. Del Debbio, M. Faber, J. Greensite and S. Olejnic, Center Dominance and $Z_{2}$ Vortices in SU(2) Lattice Gauge Theory, Phys. Rev. D55 (1997) 2298.

[7] T. T. Takahashi et al., Three-Quark Potential in SU(3) Lattice QCD, Phys. Rev. Lett. 86 (2001) 18; Detailed Analysis of the Three-Quark Potential in SU(3) Lattice QCD, Phys. Rev. D65 (2002) 114509.

[8] H. Ichie, V. G. Bornyakov, T. Streuer and G. Schierholz, Flux Tubes in Two- and Three-Quark System in Full QCD, Nucl. Phys. A721 (2003) 899; V. Bornyakov, H. Ichie et al., Baryonic Flux in Quenched and Two-Flavor Dynamical QCD after Abelian Projection, Phys. Rev. D70 (2004) 054506.

[9] H. Suganuma, A. Yamamoto, N. Sakumichi, T. T. Takahashi, H. Iida and F. Okiharu, Inter-Quark Potentials in Baryons and Multi-Quark Systems in QCD, Mod. Phys. Lett. A [arXiv:0802.3500].

[10] A. De Rujula, H. Georgi and S. L. Glashow, Hadron Masses in a Gauge Theory, Phys. Rev. D12 (1975) 147.

[11] N. I. Kochelev, Role of Instantons in Formation of Hadron Mass Spectrum, Yad. Fiz. 41 (1985) 456 [Sov. J. Nucl. Phys. 41 (1985) 291]. 